\title{
Legendre fractional differential equation and Legender fractional polynomials
}

\author{
M. Abu Hammad, R. Khalil* \\ Department of Mathematics, The University of Jordan-Amman-Jordan \\ ${ }^{*}$ Corresponding author E-mail: roshdikhalil@hotmail.com
}

Copyright (C)2014 M. Abu Hammad and R. Khalil. This is an open access article distributed under the Creative Commons Attribution License, which permits unrestricted use, distribution, and reproduction in any medium, provided the original work is properly cited.

\begin{abstract}
In this paper we study the Legender conformable fractional differential equation. It turns out that in certain cases, similar to the classical case, certain solutions are fractional polynomials. Further, we study basic properties of such fractional polynomials.
\end{abstract}

Keywords: Legendre Fractional Equation, Legendre Fractional Polynomials.

\section{Introduction}

The subject of fractional derivative is as old as calculus. In 1695, L'Hopital asked if the expression $\frac{d^{0.5}}{d x^{0.5}} f$ has any meaning. Since then, many researchers have been trying to generalize the concept of the usual derivative to fractional derivatives. These days, many definitions for the fractional derivative are available. Most of these definitions use an integral form. The most popular definitions are: by

(i) Riemann - Liouville Definition: If $n$ is a positive integer and $\alpha \in[n-1, n)$, the $\alpha^{\text {th }}$ derivative of $f$ is given

$D_{a}^{\alpha}(f)(t)=\frac{1}{\Gamma(n-\alpha)} \frac{d^{n}}{d t^{n}} \int_{a}^{t} \frac{f(x)}{(t-x)^{\alpha-n+1}} d x$.

(ii) Caputo Definition. For $\alpha \in[n-1, n)$, the $\alpha$ derivative of $f$ is

$D_{a}^{\alpha}(f)(t)=\frac{1}{\Gamma(n-\alpha)} \int_{a}^{t} \frac{f^{(n)}(x)}{(t-x)^{\alpha-n+1}} d x$.

Now, all definitions are attempted to satisfy the usual properties of the standard derivative. The only property inherited by all definitions of fractional derivative is the linearity property. However, the following are the setbacks of one definition or another:

(i) The Riemann-Liouville derivative does not satisfy $D_{a}^{\alpha}(1)=0\left(D_{a}^{\alpha}(1)=0\right.$ for the Caputo derivative), if $\alpha$ 
is not a natural number.

(ii) All fractional derivatives do not satisfy the known product rule:

$D_{a}^{\alpha}(f g)=f D_{a}^{\alpha}(g)+g D_{a}^{\alpha}(f)$.

(iii) All fractional derivatives do not satisfy the known quotient rule:

$D_{a}^{\alpha}(f / g)=\frac{g D_{a}^{\alpha}(f)-f D_{a}^{\alpha}(g)}{g^{2}}$.

(iv) All fractional derivatives do not satisfy the chain rule:

$D_{a}^{\alpha}(f \circ g)(t)=f^{(\alpha)} g(t) g^{(\alpha)}(t)$.

(v) All fractional derivatives do not satisfy: $D^{\alpha} D^{\beta} f=D^{\alpha+\beta} f$ in general

(vi) Caputo definition assumes that the function $f$ is differentiable.

(v) $T_{1}(\lambda)=0$, for all constant functions $f(t)=\lambda$.

In [ 2], a new definition called conformable fractional derivative was introduced. The new definition satisfies:

$1 . T_{\alpha}(a f+b g)=a T_{\alpha}(f)+b T_{\alpha}(g)$, for all $a, b \in R$.

$2 . T_{\alpha}(\lambda)=0$, for all constant functions $f(t)=\lambda$.

Further, for $\alpha \in(0,1]$ and and $f, g$ be $\alpha$-differentiable at a point $t$, with $g(t) \neq 0$. Then

3. $T_{\alpha}(f g)=f T_{\alpha}(g)+g T_{\alpha}(f)$.

4. $T_{\alpha}\left(\frac{f}{g}\right)=\frac{g T_{\alpha}(f)-f T_{\alpha}(g)}{g^{2}}$

We list here the fractional derivatives of certain functions, for the purpose of comparing the results of the new definition with the usual definition of the derivative:

1. $T_{\alpha}\left(t^{p}\right)=p t^{p-\alpha}$.

2. $T_{\alpha}\left(\sin \frac{1}{\alpha} t^{\alpha}\right)=\cos \frac{1}{\alpha} t^{\alpha}$.

3. $T_{\alpha}\left(\cos \frac{1}{\alpha} t^{\alpha}\right)=-\sin \frac{1}{\alpha} t^{\alpha}$.

4. $T_{\alpha}\left(e^{\frac{1}{\alpha} t^{\alpha}}\right)=e^{\frac{1}{\alpha} t^{\alpha}}$.

On letting $\alpha=1$ in these derivatives, we get the corresponding ordinary derivatives. Recently, [1], used the conformable definition of fractional derivative to introduce fractional Laplace transform, and fractional Taylor expansion. We refer to [4], [5], [6], and [7] for the basic properties and history of fractional calculus.

\section{Preliminaries}

The classical Legendre differential

$\left(1-x^{2}\right) y^{\prime \prime}-2 x y^{\prime}+k(k+1) y=0$

The point $x=0$ is an ordinary point for the equation. Solving the equation around $x=0$, using series solution, and assuming that $k$ is a natural number, gives polynomial solution, called Legender polynomials, given by the formula:

$P_{n}(x)=\frac{1}{2^{n} n !} \frac{d^{n}}{d x^{n}}\left(x^{2}-1\right)^{n}$

These sets of polynomials turned to be orthogonal polynomials in the sense $\int_{-1}^{1} P_{n}(x) P_{m}(x) d x=0$ for $n \neq m$. Such polynomials turned out to be of particular interest in many problems in mathematical physics like heat distribution in spherical regions and in the structure of atoms.

Throughout this paper, we let $D^{\alpha} y$ denote the conformable fractional derivative of $y$, where $\alpha \in(0,1]$. The second $\alpha$-derivative of $y$ will be denoted by $D^{\alpha} D^{\alpha} y$. A series is called a fractional power series if it can be 
written in the form $\sum_{n=0}^{\infty} a_{n} x^{n \alpha}$, for $\alpha \in(0,1]$. Further, [2], we let $I_{\alpha}^{0}(f) x=\int_{0}^{x} \frac{f(t)}{t^{1-\alpha}} d t$. . We refer to [1 ] and [2] for the basic structure of conformable fractional derivatives, integrals and fractional power series.

In this paper, we will be studying the fractional Legender equation: $\left(1-x^{2 \alpha}\right) D^{\alpha} D^{\alpha} y-2 \alpha x^{\alpha} D^{\alpha} y+\alpha^{2} k(k+1) y=$ 0 . We find that if $k$ is a natural number then one gets fractional polynomial solutions, to be called Legender fractional polynomials.

\section{Solution of fractional Legender equation}

Consider the equation

$\left(1-x^{2 \alpha}\right) D^{\alpha} D^{\alpha} y-2 \alpha x^{\alpha} D^{\alpha} y+\alpha^{2} k(k+1) y=0$

where $\alpha \in(0,1]$, and $k$ is any real number. Clearly, if $\alpha=1$, then equation $(*)$ is just the classical Legender equation. We will use series solution around $x=0$ to get polynomial solutions for equation $(*)$, when $k$ is assumed to be a natural number.

Now $x=0$ is an ordinary point for the equation. Using the fractional power series expansion given in [2 ], and for $x>0$, we let

$y=\sum_{n=0}^{\infty} a_{n} x^{n \alpha}$

So

$D^{\alpha} y=\sum_{n=1}^{\infty} \alpha n a_{n} x^{n \alpha-\alpha}$

$D^{\alpha} D^{\alpha} y=\sum_{n=2}^{\infty} \alpha^{2} n(n-1) a_{n} x^{n \alpha-2 \alpha}$

Substitute these in equation $(*)$ we get:

$\sum_{n=2}^{\infty} \alpha^{2} n(n-1) a_{n} x^{n \alpha-2 \alpha}-x^{2 \alpha} \sum_{n=2}^{\infty} \alpha^{2} n(n-1) a_{n} x^{n \alpha-2 \alpha}-2 \alpha x^{\alpha} \sum_{n=1}^{\infty} \alpha n a_{n} x^{n \alpha-\alpha}+\alpha^{2} k(k+1) \sum_{n=0}^{\infty} a_{n} x^{n \alpha}=0$

In the first sum in $(* *)$, replacing $n$ by $n+2$ we get

$\sum_{n=0}^{\infty} a_{n+2} \alpha^{2}(n+2)(n+1) x^{n \alpha}$

The second term sum needs no change of variables and it is equal to:

$-\sum_{n=2}^{\infty} \alpha^{2} n(n-1) a_{n} x^{n \alpha}$

The third term sum needs no change of variables and it is equal to:

$-\sum_{n=1}^{\infty} 2 \alpha^{2} n a_{n} x^{n \alpha}$ 
The forth term sum needs no change of variables and it is equal to:

$\sum_{n=0}^{\infty} \alpha^{2} k(k+1) a_{n} x^{n \alpha}$

Now, unifying all summations to start from $n=2$ and put them in one summation we get:

$$
\begin{array}{r}
\left(2 \alpha^{2} a_{2}+\alpha^{2} k(k+1) a_{0}\right)+\left(6 \alpha^{2} a_{3}-2 \alpha^{2} a_{1}+\alpha^{2} k(k+1) a_{1}\right) x \\
+\sum_{n=2}^{\infty}\left[a_{n+2} \alpha^{2}(n+2)(n+1)-\alpha^{2} n(n-1) a_{n}-2 \alpha^{2} n a_{n}+\alpha^{2} k(k+1) a_{n}\right] x^{n \alpha}=0
\end{array}
$$

From which we get:

$$
\begin{aligned}
& a_{2}=-\frac{k(k+1)}{2} a_{0} \\
& a_{3}=\frac{2-k(k+1)}{6} a_{1}
\end{aligned}
$$

and

$a_{n+2}=\frac{n(n+1)-k(k+1)}{(n+1)(n+2)} a_{n}$

Hence there are two independent solutions $y_{1}$ which is the sum over the odd terms and $y_{2}$ which is the sum over the even terms. However, such solutions do not have finite value at 1 and at -1 , which means that such solutions are not physically feasible. Thus the only series solutions of interest are those that terminate after finitely many steps. That means polynomial solutions. Thus in equation (7) if $k$ is a natural number, then $a_{k+2}=0$ and so $a_{k+2 n}=0$. However, one may still have $a_{k(2 n+1)} \neq 0$ for all $n$. Hence by choosing one of $a_{0}$ (or $a_{1}$ ) equal to zero, we can make all even-numbered coefficients (or all odd-numbered coefficients) equal to zero.

Simplifying the recurrence formula in (7), to get

$a_{n+2}=-\frac{(k-n)(n+k+1)}{(n+1)(n+2)} a_{n}$

Notice, if one substitute in the recurrence formula (8), $n=0$, we get $a_{2}=-\frac{k(k+1)}{2} a_{0}$, which is equation (5). If $n=2$, we get $a_{4}=\frac{k(k-2)(k+3)(k+1)}{4 !} a_{0}$. And son on to get

$a_{2 n}=(-1)^{n} \frac{k(k-2) \ldots(k-2(n-1)) \cdot(k+2 n-1)(k+2 n-3) \ldots(k+1)}{(2 n) !} a_{0}$

So if $k$ is an even natural number, one can simplify (9) to get

$a_{2 n}=(-1)^{n} \frac{(k+2 n) !\left[\left(\frac{k}{2}\right) !\right]^{2}}{(2 n) ! k !\left(\frac{k}{2}+n\right) !\left(\frac{k}{2}-n\right) !} a_{0}$

The constant $a_{0}$ is usually chosen so that so that the polynomial solution at $x=1$ equals 1 . So, the value to be given to $a_{0}$ is $a_{0}=(-1)^{\frac{k}{2}} \frac{k !}{2^{k}\left[\left(\frac{k}{2}\right) !\right]^{2}}$. So

for $k=0$, we get the polynomial $P_{0}(x)=1$. For $k=2$, we get the polynomial $P_{2}(x)=\frac{1}{2}\left(3 x^{2 \alpha}-1\right)$. Similarly, for $n=4,6,8, \ldots$. There are similar formulas and structure if $k$ is an odd natural number: $P_{1}(x)=x^{\alpha}$, $P_{3}(x)=\frac{1}{2}\left(5 x^{3 \alpha}-3 x^{\alpha}\right), \ldots$

Let $D^{n \alpha}=D^{\alpha} D^{\alpha} \ldots D^{\alpha}, n$-times. With such notation and using the known formula $[2], D^{\alpha} x^{p}=p x^{p-1}$, we can have a nice closed formula for all such polynomials, as follows:

$P_{n}(x)=\frac{1}{\alpha^{n} 2^{n} n !} D^{n \alpha}\left(x^{2 \alpha}-1\right)^{n}$ 


\section{Orthogonality of Legendre fractional polynomials}

In the classical sense, two functions $f, g$ are orthogonal on an interval $[a, b]$, if $\int_{a}^{b} f(x) g(x) d x=0$. For the case of Legendre polynomials, the interval that mathematicians consider is $[-1,1]$, for physical applications. So if we want to study the orthogonality of fractional Legendre polynomials on such interval, then we have to extend the definition of $D^{\alpha} f(x)$ to include negative values of $x$. In the definition given in [2], the definition was $D^{\alpha} f(t)=$ $\lim _{\varepsilon \rightarrow 0} \frac{f\left(t+\varepsilon t^{1-\alpha}\right)-f(t)}{\varepsilon}, t>0$. To avoid the trouble of being undefined on $[-1,0]$, we assume $\alpha$ to be of the form $\frac{1}{k}$, with $k$ an odd natural number. So throughout this section we assume $\alpha=\frac{1}{2 j+1}$, with $j$ any natural number. In such a case $t^{1-\alpha}$ is defined for all $t \in R$, and $x^{n \alpha}$ is defined for all $x \in R$, and all $n$. So we can put the following:

Definition 4.1. For $0<\alpha=\frac{1}{2 j+1} \leq 1$, we let $D^{\alpha} f(t)=\lim _{\varepsilon \rightarrow 0} \frac{f\left(t+\varepsilon t^{1-\alpha}\right)-f(t)}{\varepsilon}, t \neq 0$. If $t=0$, then we define $D^{\alpha} f(0)=\lim _{t \rightarrow 0} D^{\alpha} f(t)$. It follows from the definition, that for the case of fractional polynomials we have $D^{\alpha} x^{n \alpha}=n \alpha x^{(n-1) \alpha}$, for all $x \in R$.

Let $I_{\alpha}^{-1}(f)(1)=\int_{-1}^{1} \frac{f(x)}{x^{1-\alpha}} d x$. Now we can state:

Theorem 4.2. $\int_{-1}^{1} P_{n}(x) P_{m}(x) d x=0$ for $n \neq m$.

Proof. Since $P_{n}$, is a solution for the fractional equation $\left(1-x^{2 \alpha}\right) D^{\alpha} D^{\alpha} y-2 \alpha x^{\alpha} D^{\alpha} y+\alpha^{2} n(n+1) y=0$, then

$D^{\alpha}\left[\left(1-x^{2 \alpha}\right) D^{\alpha} P_{n}(x)\right]+\alpha^{2} n(n+1) P_{n}(x)=0$

Similarly for $P_{m}(x)$ :

$D^{\alpha}\left[\left(1-x^{2 \alpha}\right) D^{\alpha} P_{m}(x)\right]+\alpha^{2} m(m+1) P_{m}(x)=0$

Multiply (12) by $P_{m}$ and (13) by $P_{n}$ and subtract the resulting equation to get

$J=P_{m}(x)\left[D^{\alpha}\left[\left(1-x^{2 \alpha}\right) D^{\alpha} P_{n}(x)\right]+\alpha^{2} n(n+1) P_{n}(x)\right]-P_{n}(x)\left[D^{\alpha}\left[\left(1-x^{2 \alpha}\right) D^{\alpha} P_{m}(x)\right]+\alpha^{2} m(m+1) P_{m}(x)\right]=0$

Now, apply the fractional integral $I_{\alpha}^{-1}$ to equation (14) to get:

$$
\begin{array}{r}
I_{\alpha}^{-1}(J)=\int_{-1}^{1} \frac{J(x)}{x^{1-\alpha}} d x=0=\int_{-1}^{1} \frac{\left(P _ { m } ( x ) \left[D^{\alpha}\left[\left(1-x^{2 \alpha}\right) D^{\alpha} P_{n}(x)\right]-P_{n}(x)\left[D^{\alpha}\left[\left(1-x^{2 \alpha}\right) D^{\alpha} P_{m}(x)\right]\right)\right.\right.}{x^{1-\alpha}} d x \\
+\int_{-1}^{1} \frac{\alpha^{2}[n(n+1)-m(m+1)] P_{n}(x) P_{m}}{x^{1-\alpha}} d x=0
\end{array}
$$

Using integration by parts that was introduced in [1] for fractional derivatives, and the fact that $n \neq m$ we get the result.

\section{References}

[1] T. Abdeljawad. On conformable fractional calculus. Arxive math. To appear

[2] R. Khalil, M. Al Horani, A. Yousef, and M. Sababheh. Anew definition of fractional derivative. Journal of computational applied mathematics, 264(2014)65-70.

[3] Abu Hammad, M. and Khalil, R. Fractional Heat Equation. IJPAM. To appear. 
[4] K.S. Miller. An introduction to fractional calculus and fractional differential equations, J.Wiley, and Sons, New York 1993.

[5] K. Oldham, and J. Spanier. The fractional calculus, theory and applications of differentiation and integration of arbitrary order. Academic Press, U.S.A. 1974

[6] A.Kilbas, H. Srivastava, and J. Trujillo. Theory and applications of fractional differential equations. Math. Studies. Northholland, New York 2006.

[7] I. Podlubny. Fractional differential equations. Academic Press, U.S.A. 1999 